\title{
Cellulose as an Inert Scaffold in Plasmon-Assisted Photoregeneration of Cofactor Molecules
}

\author{
Nina Tarnowicz-Staniak, Silvia Vázquez-Díaz, Valeri Pavlov, Katarzyna Matczyszyn,* \\ and Marek Grzelczak*
}

Cite This: ACS Appl. Mater. Interfaces 2020, 12, 19377-19383

Read Online

\section{ACCESS \\ Џlll Metrics \& More \\ Article Recommendations \\ Supporting Information}

ABSTRACT: Plasmonic nanoparticles exhibit excellent light-harvesting properties in the visible spectral range, which makes them a convenient material for the conversion of light into useful chemical fuel. However, the need for using surface ligands to ensure colloidal stability of nanoparticles inhibits their photochemical performance due to the insulating molecular shell hindering the carrier transport. We show that cellulose fibers, abundant in chemical functional groups, can serve as a robust substrate for the immobilization of gold nanorods, thus also providing a facile way to remove the surfactant molecules. The resulting functional composite was implemented in a bioinspired photocatalytic process involving dehydrogenation of sodium formate and simultaneous photoregeneration of cofactor molecules $(\mathrm{NADH}$, nicotinamide adenine dinucleotide) using visible light as an energy source. By systematic screening of experimental parameters, we compare photocatalytic and thermocatalytic properties of the composite and evaluate the role of palladium cocatalyst.

KEYWORDS: cellulose, gold nanorods, palladium, plasmonic catalysis, cofactor molecules, sodium formate, photoelectrochemistry

\section{INTRODUCTION}

The development of a large assortment of plasmonic nanocrystals in terms of composition, spectral features, morphology, and sizes has facilitated multiple applications, including those of plasmonic catalysis. ${ }^{1-8}$ However, an important issue in such applications is that nanocrystals fabricated in a liquid phase require surface ligands in the form of long alkyl chain molecules to prevent undesired aggregation. Such an insulating molecular shell hinders the access of chemicals to the crystal surface, compromising its overall catalytic activity. ${ }^{9,10}$ A straightforward strategy of removing native ligands without the risk of uncontrolled aggregation is the immobilization of nanocrystals on solid substrates. Several types of substrates have been proposed so far, including $\mathrm{TiO}_{2}{ }^{10} \mathrm{CeO}_{2},{ }^{11} \mathrm{ZrO}_{2}, \mathrm{Al}_{2} \mathrm{O}_{3},{ }^{12} \mathrm{SiO}_{2},{ }^{13}$ glass, ${ }^{14-16}$ graphene, ${ }^{19}$ and $\mathrm{MoS}_{2} \cdot{ }^{18}$ The electronic properties of those materials, however, alter the dynamics of carrier flow (e.g., through a Schottky barrier), obscuring the picture of autonomous plasmonic catalysis. There is a need for inert substrates that can support autonomous plasmonic catalysis without affecting the optical features of nanoparticles, the desirable targets being organic materials of natural origin, such as cellulose.

Cellulose is an excellent scaffold candidate for the plasmonic catalyst because of its availability, chemical robustness, and high-surface area. In fact, cellulose has become an emerging material, finding applications as an adsorbent, ${ }^{19}$ flocculant, ${ }^{20,21}$ photocatalyst, $^{21,22}$ membrane, ${ }^{23,24}$ and film ${ }^{25}$ in water treatment. In particular, the combination of cellulose with semiconducting nanoparticles has been shown to produce an efficient photocatalyst for hydrogen generation ${ }^{26}$ or organic dye degradation under visible light irradiation..$^{22,27-29}$ It has also been reported that the chemically rich surface of cellulose can serve as an excellent electron donor for hydrogen generation using semiconducting nanoparticles as photocatalysts at alkaline conditions. ${ }^{30,31}$ Shape anisotropy of cellulose fibers has been exploited in templated self-assembly of plasmonic nanoparticles, leading to chiral optical properties ${ }^{32-34}$ or serving as a substrate for plasmon-based biosensing. ${ }^{33}$ Regardless of targeted applications, the integration of as-prepared anisotropic plasmonic nanoparticles within a cellulose matrix remains poorly understood because of the excess of cationic surfactants on the surface of the particles (e.g., cetyltrimethylammonium bromide, CTAB) that alter the metal-cellulose interactions. Therefore, further experimental effort is required to control cellulose-nanocrystal interactions in the presence of a surfactant to obtain a catalytically active, processable hybrid composite with well-controlled optical properties.

Received: December 10, 2019

Accepted: April 7, 2020

Published: April 7, 2020 
Recently, formic acid has attracted attention as a liquid organic hydrogen carrier due to its high gravimetric and volumetric $\mathrm{H}_{2}$ capacity, low toxicity, and ease of handling. ${ }^{35}$ Although the dehydrogenation of formic acid to $\mathrm{H}_{2}$ traditionally requires elevated temperatures and noble metal catalysts $(\mathrm{Pt}, \mathrm{Pd}$, and $\mathrm{Au})$, several photocatalytic approaches have been proposed, involving $\mathrm{N}$-modified $\mathrm{TiO}_{2},{ }^{36}$ metal-functionalized carbon nitride, ${ }^{37,38} \mathrm{CdS}$ quantum dots, ${ }^{39}$ or $\mathrm{Ag} / \mathrm{Pd}$ nanowires. ${ }^{40}$ While considering plasmonic catalysis as a useful tool for organic transformations, one has to take into account three phenomena related to excited plasmonic nanoparticles: local heating, near-field enhancement, and generation of energetic carriers. Chemical conversions can be driven either by one or by more than one of those factors, which is strictly related to the type of reactants and of nanoparticles used. The use of plasmonic catalysis in the dehydrogenation of formic acid has been reported by the group of Majima ${ }^{41}$ that suggested that charge transfer from a gold nanorod photocatalyst to the surface of a Pd cocatalyst, rather than photothermal heating, dictates the process of hydrogen formation. In another approach, Moskovits et al., ${ }^{42}$ by using a colloidal photocatalyst comprising Pd sheets, demonstrated the involvement of hot carriers in the dehydrogenation of formic acid under visible light irradiation. Although the production of molecular hydrogen directly from formic acid is technologically relevant, ${ }^{43}$ its storage arises safety and economic issues. Meanwhile, in nature, the dehydrogenation of formic acid is carried out by an enzyme known as formate dehydrogenase, which is able to transfer hydride (two electrons and a proton) directly to the cofactor molecules of nicotinamide adenine dinucleotide $\mathrm{NAD}^{+}$, leading to its reduction to $\mathrm{NADH}^{44}$ which remains chemically stable in physiological conditions ( $\mathrm{pH} 7$ ). To the best of our knowledge, there are no reports on photocatalytic regeneration of $\mathrm{NADH}$ using sodium formate as the electron donor.

Here, we show that a combination of cellulose with metal nanocrystals (Pd-coated gold nanorods) renders a composite with the photocatalytic activity in the processes of dehydrogenation of sodium formate and simultaneous reduction of $\mathrm{NAD}^{+}$ to $\mathrm{NADH}$ under visible-infrared light irradiation (Figure 1).

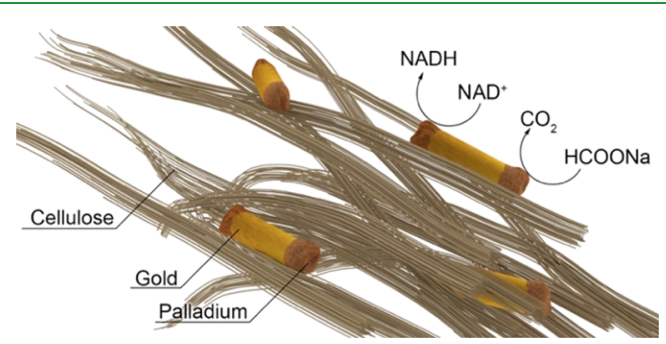

Figure 1. Schematic representation of the hybrid photocatalyst comprising Pd-coated gold nanorods (AuPdNRs) anchored to the cellulose fibers. In the presence of visible light, plasmonic nanoparticles catalyze the dehydrogenation of sodium formate and simultaneous reduction of cofactor molecules.

The fabrication of the functional composite was made possible by the rational screening of the concentrations of CTAB, nanoparticles, and cellulose to obtain a processable material in the form of gels, viscous solutions, two-phase systems, or stable colloidal solutions. Fine tuning of metal-cellulose interactions allowed us to maintain initial optical and photocatalytic properties of the palladium-gold nanocrystals even after the removal of the CTAB surfactant.

\section{EXPERIMENTAL SECTION}

2.1. Chemicals. All of the chemicals were commercially available and were used without further purification. Gold(III) chloride trihydrate $\left(\mathrm{HAuCl}_{4} \cdot 3 \mathrm{H}_{2} \mathrm{O}\right)$, potassium tetrachloropalladate(II) $\left(\mathrm{K}_{2} \mathrm{PdCl}_{4}\right)$, cetyltrimethylammonium bromide (CTAB), sodium borohydride $\left(\mathrm{NaBH}_{4}\right)$, L-ascorbic acid (AA), silver nitrate $\left(\mathrm{AgNO}_{3}\right)$, sodium hydroxide $(\mathrm{NaOH}), \beta$-nicotinamide adenine dinucleotide $\left(\mathrm{NAD}^{+}\right)$, and sodium formate $(\mathrm{HCOONa})$ were purchased from Sigma-Aldrich. Hydrochloric acid $(\mathrm{HCl}, 37 \%)$ was purchased from Scharlab. Cellulose nanofibers were supplied by CelluForce (Canada). Deionized water (Millipore Milli-Qgrade) with a resistivity of $18.2 \mathrm{M} \Omega \cdot \mathrm{cm}$ was used in all experiments.

2.2. Synthesis of Gold Nanorods (AuNRs). Gold nanorods (AuNRs) were prepared using a Ag-assisted seeded growth method. $^{45,46}$ Seeds were prepared by reduction of HAuCl4 $(0.025$ $\mathrm{mL}, 0.05 \mathrm{M})$ with ice-cold $\mathrm{NaBH} 4(0.3 \mathrm{~mL}, 0.01 \mathrm{M})$ in an aqueous $\mathrm{CTAB}$ solution $(4.7 \mathrm{~mL}, 0.1 \mathrm{M})$ under vigorous stirring. The mixture was left for $30 \mathrm{~min}$, being mildly stirred. To the growth solution containing CTAB $(10 \mathrm{~mL}, 0.1 \mathrm{M}), \mathrm{HAuCl} 4(0.1 \mathrm{~mL}, 0.05 \mathrm{M}), \mathrm{AA}$ $(0.08 \mathrm{~mL}, 0.1 \mathrm{M}), \mathrm{HCl}(0.19 \mathrm{~mL}, 1 \mathrm{M})$, and AgNO3 $(0.12 \mathrm{~mL}, 0.01$ $\mathrm{M})$, a Au seed solution $(0.024 \mathrm{~mL})$ was added. The mixture was left undisturbed at room temperature for at least $2 \mathrm{~h}$. The final concentration of metallic gold was $0.0005 \mathrm{M}$.

2.3. Preparation of Bimetallic Nanorods (AuPdNRs). Palladium overgrowth was carried out in the presence of silver anions. ${ }^{41}$ To the solution of as-synthesized AuNRs $(10 \mathrm{~mL}, 0.0005$ $\mathrm{M})$, AA ( $1.136 \mathrm{~mL}, 0.1 \mathrm{M})$ was added followed by the addition of a given amount of $\mathrm{K}_{2} \mathrm{PdCl}_{4}(0.01 \mathrm{M})$ to obtain $\mathrm{Pd}$ mol $\%$ of 10,25 , and 41.5 in relation to the Au molar content during the reduction step. Finally, $\mathrm{HCl}(80 \mu \mathrm{L}$ of $0.1 \mathrm{M})$ was added. The solution was kept at 40 ${ }^{\circ} \mathrm{C}$ for $12 \mathrm{~h}$. The samples are thereafter referred to as $\mathrm{Pd} 10,25$, and $41 \mathrm{~mol} \%$, respectively.

2.4. AuNRs-Cellulose Composite. Four solutions of asprepared bare AuNRs $(10 \mathrm{~mL}, 0.0005 \mathrm{M})$ were washed twice (9000 rpm, $15 \mathrm{~min})$ and redispersed in CTAB solutions of concentrations 15, 10,5, or $1 \mathrm{mM}$. Each solution of AuNRs was titrated with the solution containing different amounts of cellulose (5.0, 4.0, 2.5, 1.0, and $0.5 \mathrm{wt} \%)$ under vigorous stirring at room temperature, keeping a volume ratio of AuNRs/cellulose $=2: 1$. All samples were subjected to visual inspection and UV-vis-NIR characterization.

2.5. AuPdNRs-Cellulose Photocatalyst Preparation. To the solution of AuPdNRs $(2.5 \mathrm{~mL},[\mathrm{Au}]=0.00225 \mathrm{M}$, [CTAB] $=1 \mathrm{mM})$, a solution of cellulose $(1.25 \mathrm{~mL}, 0.5 \mathrm{wt} \%)$ was added dropwise under vigorous stirring. After phase separation, the mixture was centrifuged three times to remove the excess CTAB. The composite was redispersed in water $(2.5 \mathrm{~mL})$ and contained $\sim 18 \mathrm{wt} \%$ of $\mathrm{Au}$ in relation to cellulose (based on the amount of gold added).

2.6. Material Characterization. UV-vis-NIR measurements of all samples were performed using a JASCO V-770 UV-vis spectrophotometer. Transmission electron microscopy (TEM) images were acquired using a JEOL JEM-1400PLUS, and scanning TEM measurements were performed using a JEOL JEM-F200. X-ray photoelectron spectroscopy (XPS) measurements were performed using a SPECS Sage HR100 spectrometer equipped with a nonmonochromatic X-ray source (aluminum $\mathrm{K} \alpha$ line of $1486.6 \mathrm{eV}$, $300 \mathrm{~W}$ ). Thermogravimetric analysis was recorded on a TGA Q500 (TA Instruments) under nitrogen by equilibrating at $150{ }^{\circ} \mathrm{C}$ and using a ramp of $10^{\circ} \mathrm{C} / \mathrm{min}$ up to $900{ }^{\circ} \mathrm{C}$.

2.7. One-Pot Photochemical Regeneration of Cofactor Molecules. The reaction mixture $(2.5 \mathrm{~mL})$ consisting of $\mathrm{HCOONa}$ (1 M), NAD ${ }^{+}(1 \mathrm{mM})$, and a photocatalyst in a phosphate buffer $(0.1$ $\mathrm{M}$; pH 5.96 or 7.91 ) was irradiated for $2 \mathrm{~h}$ at $40{ }^{\circ} \mathrm{C}$ using Fiber-Lite MI-150 light source (Dolan-Jenner Industries). The light power density was $200 \mathrm{~mW} / \mathrm{cm}^{2}$, and the spectral range was $400-1200 \mathrm{~nm}$. The NADH concentration was determined based on the UV-vis- 
NIR absorption spectra measurements. An aliquot collected every 30 min was put directly to the $1 \mathrm{~mm}$ quartz cuvette for measurements. After $2 \mathrm{~h}$ of irradiation, the whole sample was centrifuged $(3000 \mathrm{rpm}$, $15 \mathrm{~min}$ ), and the supernatant was collected from above the precipitate to determine the absolute amount of regenerated NADH.

2.8. Photoelectrochemical Measurements. The photocurrent measurements were conducted on DRP-110 (DropSens) at $-0.4 \mathrm{~V}$ (versus $\mathrm{Ag}$ ) by a potentiostat (Autolab Electrochemical Workstation, PGSTAT302N) using carbon and $\mathrm{Ag}$ as the counter and pseudoreference electrode, respectively. The working electrodes were functionalized by drop-casting the solutions $(15 \mu \mathrm{L})$ of either bare cellulose or AuNRs-cellulose or AuPdNRs-cellulose onto the working electrode followed by the evaporation at room temperature overnight. For all photoelectrochemical experiments, sodium formate $(20 \mu \mathrm{L}, 1 \mathrm{M})$ was deposited on the electrode surface followed by a current stabilization time of $60 \mathrm{~s}$. All analyses were performed at room temperature. The incident light source was a halogen illuminator (Fiber-Lite MI-150, Dolan-Jenner Industries) with a spectral range of $400-1200 \mathrm{~nm}$ and a power density on the sample of $87 \mathrm{~mW} / \mathrm{cm}^{2}$.

\section{RESULTS AND DISCUSSION}

The interaction of negatively charged cellulose fibers with CTAB molecules leads to the formation of gels or suspensions whose final composition depends on the concentrations of each component. ${ }^{47}$ Since $\mathrm{CTAB}$ molecules are an intrinsic component of the colloidal dispersion of anisotropic gold nanoparticles, the attempt at immobilization of as-prepared nanocrystals on cellulose fibers requires an excess of a surfactant (at least at its critical micelle concentration, 1 $\mathrm{mM}$ ), which may result in the formation of unpredictable phases. To ensure maximal loading of nanoparticles on the cellulose matrix, we screened systematically experimental conditions such as the concentration of CTAB $(1-15 \mathrm{mM})$ and cellulose $(0.5-5 \mathrm{wt} \%)$ at a fixed concentration of bare gold nanorods (length $=33.6 \pm 4.8 \mathrm{~nm}$, width $=9.3 \pm 1.3 \mathrm{~nm}$ ) (Figure 2a and Figure S1). We found that the formation of a stable, transparent gel and a viscous solution takes place at a high concentration of cellulose and low concentration of CTAB (Figure 2a, red and pink). On the contrary, a stable colloidal solution was observed with the decrease of cellulose concentration and the increase of surfactant concentration (Figure 2a, blue). Presumably, either the free surfactant molecules interact with cellulose, hindering the attachment of gold nanoparticles, or the excess surfactant stabilizes the dispersion of cellulose fibers that are completely covered with nanocrystals. Nevertheless, at the optimal conditions, phase separation occurs upon mixing the nanorods and cellulose fibers (Figure 2a, clear blue). Thereby, a clear supernatant remaining over the solid precipitate indicates complete anchoring of nanorods to the fibers. Therefore, for further studies, we selected the experimental conditions corresponding to the clear blue area (phase separation) in the phase diagram.

To confirm that the optical properties of our hybrid system stem from the presence of gold nanorods and not from the scattering contribution from the cellulose substrate, we performed a quantitative spectroscopy analysis of each sample, estimating the intensity ratio of longitudinal and transverse surface plasmon bands (Figure $2 \mathrm{~b}$ and Figure S2). We found that the intensity ratio between two plasmon bands increases with decreasing amounts of both cellulose and CTAB at constant gold nanorod loading. Therefore, we set the values of $1 \mathrm{mM}$ and 0.5 wt $\%$ as the optimal CTAB and cellulose concentrations, respectively. To maximize the gold content within the hybrid material without altering optical properties, we prepared a set of samples with a constant volume ratio of

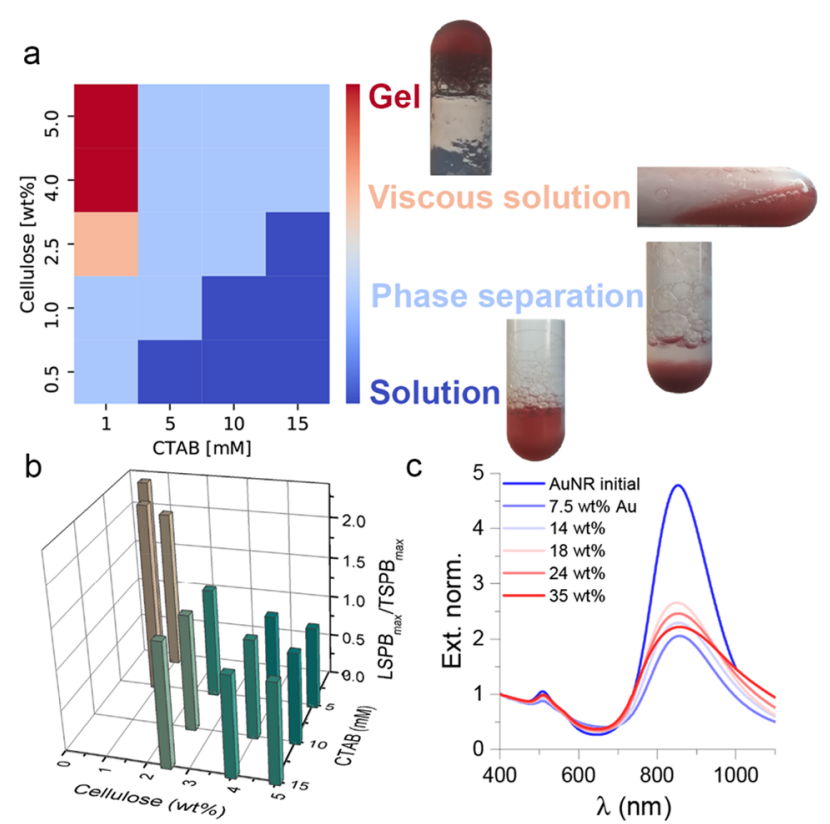

Figure 2. AuNRs-cellulose (2:1, v/v) composite. (a) Quasi-phase diagram of the system as a function of both CTAB and cellulose concentrations at a constant Au loading. Representative digital images of four identified phases: gel, viscous solution, two-phase system, and stable solution. (b) Quantitative optical analysis of two-phase systems (clear blue in panel (a)) showing that plasmon band contribution to the overall optical properties of the material is substantial only at low $\mathrm{CTAB}$ and cellulose concentrations. (c) UV-vis-NIR spectra of the two-phase systems with different $\mathrm{Au}$ mass contents (wt \%) in relation to the dry mass of cellulose, showing a broadening of the longitudinal plasmon band above 18 wt \% of Au.

AuNRs/cellulose $(2: 1 \mathrm{v} / \mathrm{v})$, fixed CTAB $(1 \mathrm{mM})$, and cellulose (0.5 wt \%) concentrations (Figure $2 c$ and Figure S3). The analysis of optical properties shows that above $18 \mathrm{wt} \% \mathrm{Au}$, the plasmon band intensity decreases, which is accompanied by its broadening. Presumably, the reason for such behavior is plasmon coupling, originating from small interparticle distances. ${ }^{48}$ Therefore, to avoid the effect of plasmon coupling on the photocatalytic process, we set 18 wt \% of $\mathrm{Au}$ as the upper limit.

Subsequently, we extended the optimized conditions for the incorporation of plasmonic nanoparticles into the cellulose matrix to the Pd-coated gold nanorods (AuPdNRs). The overgrowth of nanorods with $\mathrm{Pd}$ was performed according to the previously published method. ${ }^{41}$ Optical characterization confirmed the reduction of $\mathrm{Pd}$ on the gold surface. With an increasing amount of $\mathrm{Pd}(10,25$, and $41 \mathrm{~mol} \%)$, the plasmon band was progressively damped and broadened, which is due to the higher values of the imaginary parts of the dielectric function of palladium as compared to gold (Figure 3a). ${ }^{49}$ Despite the decrease of the longitudinal plasmon band intensity, the absorption coefficients are unaffected, which results in the reduced re-emission of photon. ${ }^{50}$ The presence of $\mathrm{Pd}$ on the surface of the nanoparticles was confirmed by scanning transmission electron microscopy (STEM) and energy dispersion spectroscopy (EDS) elemental maps (Figure S4). The characterization of the composite comprising cellulose and AuPd nanoparticles by low-resolution TEM confirmed the attachment of the nanoparticles to the fibers since the nanoparticles follow the direction of dry fibers on the TEM grid, and no free AuPdNRs were found apart from the 
a

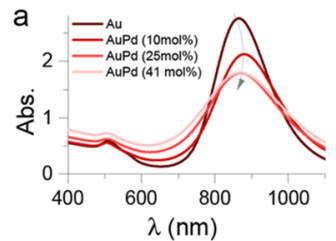

C

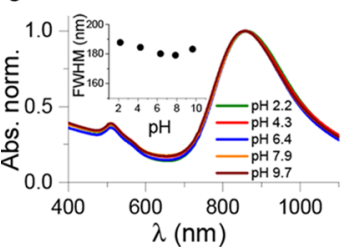

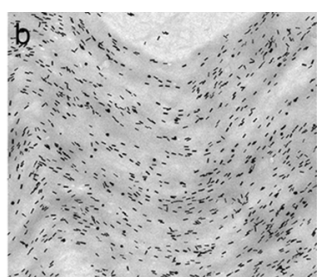
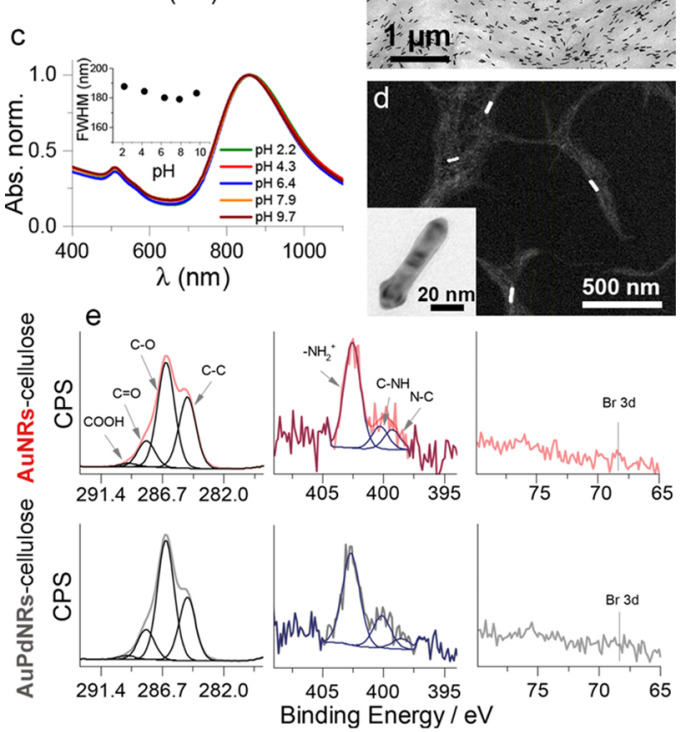

Figure 3. AuNRs-cellulose and AuPdNRs-cellulose composites. (a) UV-vis-NIR spectra of AuNRs coated with different Pd contents. (b) TEM image of AuPdNRs attached to the cellulose fibers. (c) UV-vis-NIR spectra of the composite at different $\mathrm{pH}$. Inset: FWHM values of the longitudinal surface plasmon bands at different $\mathrm{pH}$ on the corresponding spectra. (d) STEM image (dark mode) of the composite with visible cellulose. Inset: TEM image of individual AuPdNR coated with Pd (Pd $41 \mathrm{~mol} \%)$. (e) Photoemission spectra and fit of C 1s (left column) and N 1s (middle column) for AuNRscellulose (upper row) and AuPdNRs-cellulose (lower row). In both composites, the native surfactant is removed since no $\mathrm{Br}$ was detected (right column).

cellulose fibers. (Figure 3b). STEM analysis further confirmed the selective attachment of the nanocrystals to the fibers (Figure 3d). It is worth mentioning that the composite exhibits good structural integrity over a wide range of $\mathrm{pH}(2-10)$ (Figure 3c). Finally, X-ray photoelectron spectroscopy (XPS) revealed the presence of $\mathrm{Au}$ and both $\mathrm{Au}$ and $\mathrm{Pd} / \mathrm{PdO}$ in AuNRs-cellulose and AuPdNRs-cellulose samples, respectively (Figure S5). In addition, the high-resolution XPS (Figure $3 \mathrm{e})$ confirmed the presence of various functional chemical groups in the composites (namely $\mathrm{COOH}, \mathrm{C}-\mathrm{O}, \mathrm{C}=\mathrm{O}$, and $\mathrm{C}-\mathrm{NH}$ ) that originate from cellulose and indicate the abundance of anchor points for metallic nanoparticles. Such a statement is further confirmed by the absence of $\mathrm{Br} 3 \mathrm{~d}$ peaks at $68.7 \mathrm{eV}$ in both AuNRs-cellulose and AuPdNRs-cellulose composites (Figure 3e, right column). This confirms that after the immobilization and subsequent washing, the CTAB is removed from nanoparticles, thereby making the metal surface accessible for photochemical processes.

The exposure of the AuPdNRs-cellulose composite to the white light $\left(400-1200 \mathrm{~nm}, 200 \mathrm{~mW} / \mathrm{cm}^{2}\right)$ in the presence of sodium formate $(1 \mathrm{M})$ led to the gradual conversion of $\mathrm{NAD}^{+}$ $(1 \mathrm{mM})$ to $\mathrm{NADH}$, corroborating our central hypothesis of direct cofactor reduction using sodium formate as an electron donor (Figure 4). The qualitative analysis of produced NADH over time was obtained by monitoring the absorbance at 340
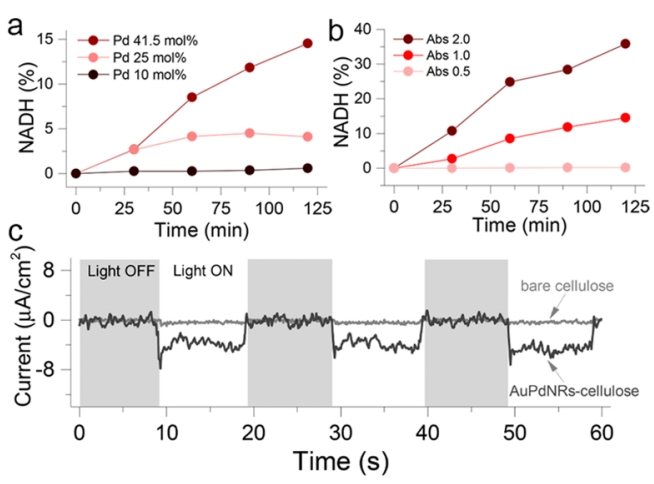

Figure 4. Photoregeneration of NADH under visible light and the effect of cellulose on the photocatalytic performance. (a) Effect of the $\mathrm{Pd}$ content and (b) composite concentration on the photoregeneration of NADH. (c) Photocurrent response at $-0.4 \mathrm{~V}$ versus the $\mathrm{Ag}$ electrode and $1 \mathrm{M}$ sodium formate under periodic on/off visible-NIR light $(400-1200 \mathrm{~nm})$ of bare cellulose compared with AuPdNRs-cellulose.

$\mathrm{nm}$ (molar absorption coefficient, $6220 \mathrm{M}^{-1} \mathrm{~cm}^{-1}$ ). The presence of metallic Pd on gold nanorods was critical for the overall photocatalytic process since no $\mathrm{NADH}$ formation was observed on the bare gold nanorods (Figure S6). Nevertheless, we observed that with the increasing Pd amount (from Pd 10 to $\mathrm{Pd} 41 \mathrm{~mol} \%)$, the rate of $\mathrm{NADH}$ production increased (Figure 4a). Similarly, with an increasing amount of the composite (measured as absorbance at the maximum of the longitudinal plasmon band), the conversion rate of $\mathrm{NADH}$ increased, reaching a maximum conversion of $40 \%$ at $\mathrm{Abs}_{\mathrm{LSPR}}=$ 2 (Figure $4 \mathrm{~b}$ ). The rate of NADH reduction is also $\mathrm{pH}$ dependent. That is, at $\mathrm{pH} 8$, the amount of regenerated NADH after $2 \mathrm{~h}$ of irradiation was five times higher compared to the reaction performed at $\mathrm{pH} 6$ (Figure S7). It has been reported that dehydrogenation of sodium formate to molecular hydrogen on a Pd electrode is the most efficient at the value of pKa of formic acid $(\sim 4) .{ }^{51}$ On the other hand, it has been shown in other studies that NADH molecules exhibit better stability at $\mathrm{pH}>7$ since at low $\mathrm{pH}, \mathrm{NADH}$ oxidizes back to $\mathrm{NAD}^{+} .52$ Therefore, while at a lower $\mathrm{pH}$, the dehydrogenation of sodium formate can be favored, the back oxidation of asreduced $\mathrm{NADH}$ molecules at a low $\mathrm{pH}$ can diminish the overall performance of the process. This suggests that the overall efficiency of the process depends on the stability of the end product $(\mathrm{NADH})$ rather than the rate of formic acid dehydrogenation. Finally, under visible light, bare cellulose shows no photocurrent density at $-0.4 \mathrm{~V}$ versus the $\mathrm{Ag}$ electrode as compared to the photocurrent density of AuPdNRs-cellulose. This leads to an important conclusion that cellulose is an inert component in the plasmonic catalysis (Figure 4c).

It is commonly known that dehydrogenation of sodium formate to molecular hydrogen on palladium as the catalyst can lead to the formation of palladium hydride, which inhibits hydrogen evolution. ${ }^{42}$ Similarly, it is known that the reduction of $\mathrm{NAD}^{+}$to NADH can proceed in the presence of molecular hydrogen. ${ }^{53}$ Therefore, we asked to what extent both processes (dehydrogenation of sodium formate and $\mathrm{NAD}^{+}$reduction) are surface dependent. In a first control experiment, a sample containing a photocatalyst ( $\mathrm{Pd} 41 \mathrm{~mol} \%)$, sodium formate (1 $\mathrm{M}, \mathrm{pH} 8)$, and $\mathrm{NAD}^{+}(1 \mathrm{mM})$ was exposed to visible light for $1 \mathrm{~h}$ followed by monitoring of NADH evolution in the dark (Figure 5a and Figure S8a). We observed a gradual increase of 

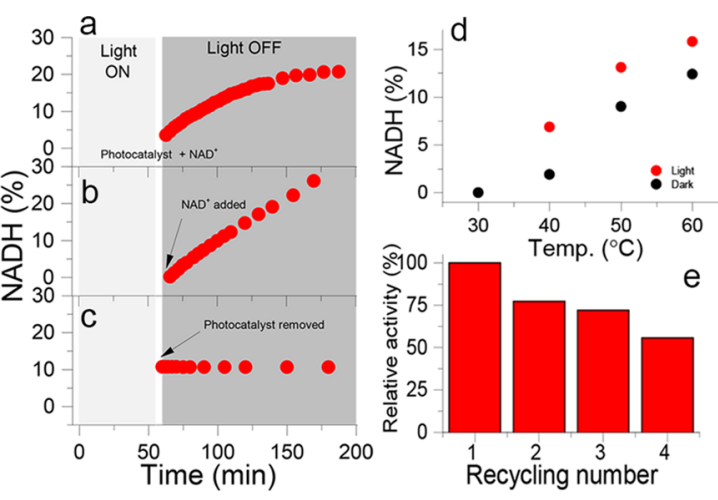

Figure 5. Control experiments, photocatalysis versus thermocatalysis, and material reusability. (a) Reduction of NADH in the dark preceded by irradiation of the mixture $\left(1 \mathrm{M}\right.$ formate, $1 \mathrm{mM} \mathrm{NAD}^{+}$, and AuPdNRs-cellulose). (b) Reduction of freshly added $\mathrm{NAD}^{+}$to the pre-irradiated mixture ( $1 \mathrm{M}$ formate and AuPdNRs-cellulose). (c) No NADH reduction in the dark after pre-irradiation of the mixture ( $1 \mathrm{M}$ formate, $1 \mathrm{mM} \mathrm{NAD}{ }^{+}$, and AuPdNRs-cellulose) and removal of AuPdNRs-cellulose. (d) Effect of temperature on NADH regeneration under dark and light conditions. (e) Reusability of the composite in $\mathrm{NADH}$ photoregeneration.

$\mathrm{NADH}$ concentration, suggesting that light induces the accumulation of species capable of reducing $\mathrm{NAD}^{+}$to $\mathrm{NADH}$ in the dark. These species can be palladium hydride or temporally reduced surface functional groups of cellulose. The accumulation of reductive species was further confirmed in another control experiment in which the photocatalytic mixture containing a photocatalyst and sodium formate (no $\mathrm{NAD}^{+}$) was pre-irradiated for $1 \mathrm{~h}$ and followed by the addition of $\mathrm{NAD}^{+}$. A reduction of $\mathrm{NAD}^{+}$to $\mathrm{NADH}$ was recorded (Figure $5 \mathrm{~b}$ and Figure $\mathrm{S} 8 \mathrm{~b}$ ). To exclude an eventual contribution of molecular $\mathrm{H}_{2}$ in $\mathrm{NADH}$ reduction, we removed AuPdNRs-cellulose by centrifugation from the photocatalytic mixture containing sodium formate and $\mathrm{NAD}^{+} / \mathrm{NADH}$ once the light was switched off. No further NADH reduction was observed in the resulting supernatant (Figure $5 \mathrm{c}$ and Figure S8c). All the above results suggest that the dehydrogenation of sodium formate and the reduction of $\mathrm{NAD}^{+}$to $\mathrm{NADH}$ are surface dependent. Importantly, the electron-donating character of cellulose can be ruled out since no reduction of $\mathrm{NAD}^{+}$to $\mathrm{NADH}$ was observed in the absence of sodium formate (Figure S9).

Since catalytic dehydrogenation of sodium formate requires elevated temperatures, thermal and photocatalytic contributions to the process of NADH regeneration were evaluated. The reduction of $\mathrm{NAD}^{+}$to $\mathrm{NADH}$ in the dark, in the presence of the photocatalyst, increases with increasing temperature: while no $\mathrm{NADH}$ is observed at $30^{\circ} \mathrm{C}$, nearly $12 \%$ was detected at $60{ }^{\circ} \mathrm{C}$ (Figure $5 \mathrm{~d}$ ). The presence of light (within the same temperature range) additionally increases the amount of regenerated $\mathrm{NADH}$. However, the ratios of $\mathrm{NADH}$ reduced during irradiation to $\mathrm{NADH}$ reduced in the dark decrease from 3.7 to 1.4 to 1.2 as the temperature increases from 40 to 50 to $60{ }^{\circ} \mathrm{C}$, respectively, suggesting the decreasing contribution of the plasmonic effect with the increase of temperature. Nevertheless, a higher ratio of $\mathrm{NADH}_{\text {light }}$ to $\mathrm{NADH}_{\text {dark }}$ at 40 ${ }^{\circ} \mathrm{C}$ indicates the inherent contribution of the plasmonic effect. Our results are consistent with the observation by Majima and co-workers ${ }^{41}$ who attributed the hydrogen evolution from formic acid on Pd-coated gold nanorods to the transfer of carriers from the metallic gold to the palladium shell.

Since AuPdNRs immobilized on cellulose matrix are easy to handle, the photocatalytic performance in four consecutive runs was tested, showing that the material preserves photocatalytic activity (Figure 5e and Figure S10). The drop to nearly $60 \%$ of its initial efficiency suggests a progressive inhibition of the metal surface by the cellulose fibers. It is conceivable that both chemically rich surface and the dynamic structure of cellulose can cause a gradual embedment of the plasmonic nanocrystal within the scaffold matrix upon the exposure to harsh chemical environments during the photochemical process. Finally, while the presence of extra CTAB is needed to homogeneously distribute nanoparticles on cellulose fibers (Figure 2), the presence of extra surfactant molecules in the AuPdNRs-composite inhibits completely the photocatalytic activity of the material (Figure S11). Thus, it has to be emphasized yet again that the benefit of using the cellulose scaffold lies in the possibility of eliminating the surfactant molecules during postsynthetic processing without altering the optical properties of the nanoparticles and in enabling the occurrence of surface processes.

\section{CONCLUSIONS}

We showed a conceptually new plasmon-based photochemical process in which surface plasmon resonance drives the dehydrogenation of sodium formate and simultaneous reduction of cofactor molecules, reaching up to $40 \%$ of conversion without the need of using molecular mediators. The surface of Pd-coated gold nanorods catalyzes the simultaneous dehydrogenation of formic acid and reduction of the cofactor as a photocatalyst. The surface photocatalytic process in the colloidal phase was made possible by anchoring plasmonic nanoparticles onto cellulose fibers, which played the role of an inert scaffold after the removal of the native surfactant molecules. The nanoparticles-cellulose hybrid photocatalytic system was obtained by a rational screening of the concentrations of each component (plasmonic nanoparticles, surfactant, and cellulose), enabling thus the fabrication of a wide variety of composites of different phases, including gel, viscous liquid, two-phase system, or stable colloidal solution. We foresee that with the functionality demonstrated here, the plasmonic nanocrystals-cellulose hybrid system can serve as a starting point toward processable macroscopic materials with hierarchical structuring employing of $3 \mathrm{D}$ printing technology. ${ }^{54}$

\section{ASSOCIATED CONTENT}

\section{Supporting Information}

The Supporting Information is available free of charge at https://pubs.acs.org/doi/10.1021/acsami.9b21556.

TEM image and histograms of bare gold nanorods, UVvis-NIR spectra of AuNRs-cellulose composites, TGA analysis results, EDS elemental maps, XPS analysis results, effect of $\mathrm{pH}$ on the photoregeneration of $\mathrm{NADH}$, UV-vis spectra of the samples during investigations of the mechanism of cellulose-assisted photocatalysis, UVvis spectra of the photocatalyst after each recycling cycle, and UV-vis spectra of all of the control experiments (PDF) 


\section{AUTHOR INFORMATION}

\section{Corresponding Authors}

Katarzyna Matczyszyn - Wroctaw University of Science and Technology, Advanced Materials Engineering and Modelling Group, 50-370 Wroctaw, Poland; (1) orcid.org/0000-00018578-8340; Email: katarzyna.matczyszyn@pwr.edu.pl

Marek Grzelczak - Centro de Fisica de Materiales CSIC-UPV/ EHU and Donostia International Physics Center DIPC, 20018 Donostia-San Sebastián, Spain; Ikerbasque, Basque Foundation for Science, 48013 Bilbao, Spain; 이이. orcidorg-0000-00023458-8450; Email: marek.grzelczak@dipc.org

\section{Authors}

Nina Tarnowicz-Staniak - Wroctaw University of Science and Technology, Advanced Materials Engineering and Modelling Group, 50-370 Wroctaw, Poland

Silvia Vázquez-Díaz - CIC biomaGUNE, 20014 Donostia-San Sebastián, Spain

Valeri Pavlov - CIC biomaGUNE, 20014 Donostia-San Sebastián, Spain; 이이.org/0000-0002-5885-9710

Complete contact information is available at: https://pubs.acs.org/10.1021/acsami.9b21556

\section{Notes}

The authors declare no competing financial interest.

\section{ACKNOWLEDGMENTS}

M.G. acknowledges funding from the Spanish MINECO (grant MAT2013-49375-EXP) and the BBVA Foundation "Primera convocatoria de ayudas fundacion BBVA a investigadores, innovadores y creadores culturales". N.T.-S. acknowledges the support from the BioTechNan (NCBiR) program of interdisciplinary $\mathrm{PhD}$ studies at Wrocław University of Science and Technology as well as the support from the Photonics and Bionanotechnology Association (PhoBiA). The work was also financed by a statutory activity subsidy from the Polish Ministry of Science and Higher Education for the Faculty of Chemistry of Wrocław University of Science and Technology. S.V. D. and V. P. acknowledge the support by the Spanish Ministry of Science, Innovation, and Universities (project BIO2017-88030-R), Maria de Maeztu Units of Excellence Program from the Spanish State Research Agency grant no. MDM-2017-0720. Authors would like to acknowledge the help from Ana Sánchez-Iglesias, Andrzej Żak, and Silvia Arrese-Igor Irigoyen, as well as JEOL Ltd. for making microscopes accessible.

\section{REFERENCES}

(1) Zhan, C.; Chen, X.-J.; Yi, J.; Li, J.-F.; Wu, D.-Y.; Tian, Z.-Q. From Plasmon-Enhanced Molecular Spectroscopy to PlasmonMediated Chemical Reactions. Nat. Rev. Chem. 2018, 2, 216-230.

(2) Gellé, A.; Jin, T.; de la Garza, L.; Price, G. D.; Besteiro, L. V.; Moores, A. Applications of Plasmon-Enhanced Nanocatalysis to Organic Transformations. Chem. Rev. 2020, 120, 986-1041.

(3) Zhou, N.; López-Puente, V.; Wang, Q.; Polavarapu, L.; Pastoriza-Santos, I.; Xu, Q.-H. Plasmon-Enhanced Light Harvesting: Applications in Enhanced Photocatalysis Photodynamic Therapy and Photovoltaics. RSC Adv. 2015, 5, 29076-29097.

(4) Kazuma, E.; Kim, Y. Mechanistic Studies of Plasmon Chemistry on Metal Catalysts. Angew. Chem. Int. Ed. 2019, 58, 4800-4808.

(5) Furube, A.; Hashimoto, S. Insight into Plasmonic Hot-Electron Transfer and Plasmon Molecular Drive: New Dimensions in Energy Conversion and Nanofabrication. NPG Asia Mater. 2017, 9, No. e454.
(6) Aslam, U.; Rao, V. G.; Chavez, S.; Linic, S. Catalytic Conversion of Solar to Chemical Energy on Plasmonic Metal Nanostructures. Nat. Catal. 2018, 1, 656-665.

(7) Zhang, X.; Ke, X.; Yao, J. Recent Development of PlasmonMediated Photocatalysts and Their Potential in Selectivity Regulation. J. Mater. Chem. A 2018, 6, 1941-1966.

(8) Kim, M.; Lin, M.; Son, J.; Xu, H.; Nam, J.-M. Hot-ElectronMediated Photochemical Reactions: Principles, Recent Advances, and Challenges. Adv. Opt. Mater. 2017, 5, 1700004.

(9) Zheng, Z.; Tachikawa, T.; Majima, T. Single-Particle Study of PtModified Au Nanorods for Plasmon-Enhanced Hydrogen Generation in Visible to Near-Infrared Region. J. Am. Chem. Soc. 2014, 136, 6870-6873.

(10) Liu, L.; Ouyang, S.; Ye, J. Gold-Nanorod-Photosensitized Titanium Dioxide with Wide-Range Visible-Light Harvesting Based on Localized Surface Plasmon Resonance. Angew. Chem. Int. Ed. 2013, 52, 6689-6693.

(11) Ke, X.; Zhang, X.; Zhao, J.; Sarina, S.; Barry, J.; Zhu, H. Selective Reductions Using Visible Light Photocatalysts of Supported Gold Nanoparticles. Green Chem. 2013, 15, 236-244.

(12) Christopher, P.; Xin, H.; Linic, S. Visible-Light-Enhanced Catalytic Oxidation Reactions on Plasmonic Silver Nanostructures. Nat. Chem. 2011, 3, 467-472.

(13) Mukherjee, S.; Zhou, L.; Goodman, A. M.; Large, N.; AyalaOrozco, C.; Zhang, Y.; Nordlander, P.; Halas, N. J. Hot-ElectronInduced Dissociation of $\mathrm{H}_{2}$ on Gold Nanoparticles Supported on $\mathrm{SiO}_{2}$. J. Am. Chem. Soc. 2014, 136, 64-67.

(14) Mubeen, S.; Lee, J.; Liu, D.; Stucky, G. D.; Moskovits, M. Panchromatic Photoproduction of $\mathrm{H}_{2}$ with Surface Plasmons. Nano Lett. 2015, 15, 2132-2136.

(15) Sánchez-Iglesias, A.; Chuvilin, A.; Grzelczak, M. PlasmonDriven Photoregeneration of Cofactor Molecules. Chem. Commun. 2015, 51, 5330-5333.

(16) Sánchez-Iglesias, A.; Barroso, J.; Solís, D. M.; Taboada, J. M.; Obelleiro, F.; Pavlov, V.; Chuvilin, A.; Grzelczak, M. Plasmonic Substrates Comprising Gold Nanostars Efficiently Regenerate Cofactor Molecules. J. Mater. Chem. A 2016, 4, 7045-7052.

(17) Kumar, D.; Lee, A.; Lee, T.; Lim, M.; Lim, D.-K. Ultrafast and Efficient Transport of Hot Plasmonic Electrons by Graphene for Pt Free, Highly Efficient Visible-Light Responsive Photocatalyst. Nano Lett. 2016, 16, 1760-1767.

(18) Zhang, P.; Fujitsuka, M.; Majima, T. Hot Electron-Driven Hydrogen Evolution Using Anisotropic Gold Nanostructure Assembled Monolayer $\mathrm{MoS}_{2}$. Nanoscale 2017, 9, 1520-1526.

(19) Qiao, H.; Zhou, Y.; Yu, F.; Wang, E.; Min, Y.; Huang, Q.; Pang, L.; Ma, T. Effective Removal of Cationic Dyes Using CarboxylateFunctionalized Cellulose Nanocrystals. Chemosphere 2015, 141, 297303.

(20) Yu, H.-Y.; Zhang, D.-Z.; Lu, F.-F.; Yao, J. New Approach for Single-Step Extraction of Carboxylated Cellulose Nanocrystals for Their Use As Adsorbents and Flocculants. ACS Sustainable Chem. Eng. 2016, 4, 2632-2643.

(21) Shak, K. P. Y.; Pang, Y. L.; Mah, S. K. Nanocellulose: Recent Advances and Its Prospects in Environmental Remediation. Beilstein J. Nanotechnol. 2018, 9, 2479-2498.

(22) Tu, K.; Wang, Q; Lu, A.; Zhang, L. Portable Visible-Light Photocatalysts Constructed from $\mathrm{Cu}_{2} \mathrm{O}$ Nanoparticles and Graphene Oxide in Cellulose Matrix. J. Phys. Chem. C 2014, 118, 7202-7210.

(23) Cruz-Tato, P.; Ortiz-Quiles, E. O.; Vega-Figueroa, K.; SantiagoMartoral, L.; Flynn, M.; Díaz-Vázquez, L. M.; Nicolau, E. Metalized Nanocellulose Composites as a Feasible Material for Membrane Supports: Design and Applications for Water Treatment. Environ. Sci. Technol. 2017, 51, 4585-4595.

(24) Karim, Z.; Mathew, A. P.; Grahn, M.; Mouzon, J.; Oksman, K. Nanoporous Membranes with Cellulose Nanocrystals as Functional Entity in Chitosan: Removal of Dyes from Water. Carbohydr. Polym. 2014, 112, 668-676.

(25) Suopajärvi, T.; Koivuranta, E.; Liimatainen, H.; Niinimäki, J. Flocculation of Municipal Wastewaters with Anionic Nanocelluloses: 
Influence of Nanocellulose Characteristics on Floc Morphology and Strength. J. Environ. Chem. Eng. 2014, 2, 2005-2012.

(26) An, X.; Wen, Y.; Almujil, A.; Cheng, D.; Li, J.; Jia, X.; Zou, J.; $\mathrm{Ni}$, Y. Nano-Fibrillated Cellulose (NFC) as Versatile Carriers of $\mathrm{TiO}_{2}$ Nanoparticles (TNPs) for Photocatalytic Hydrogen Generation. RSC Adv. 2016, 6, 89457-89466.

(27) Lefatshe, K.; Muiva, C. M.; Kebaabetswe, L. P. Extraction of Nanocellulose and In-Situ Casting of $\mathrm{ZnO} /$ Cellulose Nanocomposite with Enhanced Photocatalytic and Antibacterial Activity. Carbohydr. Polym. 2017, 164, 301-308.

(28) Anirudhan, T. S.; Deepa, J. R. Nano-Zinc Oxide Incorporated Graphene Oxide/Nanocellulose Composite for the Adsorption and Photo Catalytic Degradation of Ciprofloxacin Hydrochloride from Aqueous Solutions. J. Colloid Interface Sci. 2017, 490, 343-356.

(29) Tian, C.; Luo, S.; She, J.; Qing, Y.; Yan, N.; Wu, Y.; Liu, Z. Cellulose Nanofibrils Enable Flower-like BiOCl for High-Performance Photocatalysis under Visible-Light Irradiation. Appl. Surf. Sci. 2019, 464, 606-615.

(30) Kuehnel, M. F.; Reisner, E. Solar Hydrogen Generation from Lignocellulose. Angew. Chem. Int. Ed. 2018, 57, 3290-3296.

(31) Wakerley, D. W.; Kuehnel, M. F.; Orchard, K. L.; Ly, K. H.; Rosser, T. E.; Reisner, E. Solar-Driven Reforming of Lignocellulose to $\mathrm{H}_{2}$ with a CdS/CdO ${ }_{x}$ Photocatalyst. Nat. Energy 2017, 2, 17021.

(32) Lagerwall, J. P. F.; Schütz, C.; Salajkova, M.; Noh, J.; Hyun Park, J.; Scalia, G.; Bergström, L. Cellulose Nanocrystal-Based Materials: From Liquid Crystal Self-Assembly and Glass Formation to Multifunctional Thin Films. NPG Asia Mater. 2014, 6, No. e80.

(33) Van Rie, J.; Thielemans, W. Cellulose-Gold Nanoparticle Hybrid Materials. Nanoscale 2017, 9, 8525-8554.

(34) Querejeta-Fernández, A.; Kopera, B.; Prado, K. S.; Klinkova, A.; Methot, M.; Chauve, G.; Bouchard, J.; Helmy, A. S.; Kumacheva, E. Circular Dichroism of Chiral Nematic Films of Cellulose Nanocrystals Loaded with Plasmonic Nanoparticles. ACS Nano 2015, 9, 1037710385.

(35) Wang, X.; Meng, Q.; Gao, L.; Jin, Z.; Ge, J.; Liu, C.; Xing, W. Recent Progress in Hydrogen Production from Formic Acid Decomposition. Int. J. Hydrogen Energy 2018, 43, 7055-7071.

(36) Halasi, G.; Schubert, G.; Solymosi, F. Photolysis of HCOOH over Rh Deposited on Pure and N-Modified $\mathrm{TiO}_{2}$. Catal. Lett. 2012, 142, 218-223.

(37) Cai, Y.-Y.; Li, X.-H.; Zhang, Y.-N.; Wei, X.; Wang, K.-X.; Chen, J.-S. Highly Efficient Dehydrogenation of Formic Acid over a Palladium-Nanoparticle-Based Mott-Schottky Photocatalyst. Angew. Chem. Int. Ed. 2013, 52, 11822-11825.

(38) Xiao, L.; Jun, Y.-S.; Wu, B.; Liu, D.; Chuong, T. T.; Fan, J.; Stucky, G. D. Carbon Nitride Supported AgPd Alloy Nanocatalysts for Dehydrogenation of Formic Acid under Visible Light. J. Mater. Chem. A 2017, 5, 6382-6387.

(39) Kuehnel, M. F.; Wakerley, D. W.; Orchard, K. L.; Reisner, E. Photocatalytic Formic Acid Conversion on CdS Nanocrystals with Controllable Selectivity for $\mathrm{H}_{2}$ or CO. Angew. Chem. Int. Ed. 2015, 54, 9627-9631.

(40) Liu, H.; Liu, X.; Yu, Y.; Yang, W.; Li, J.; Feng, M.; Li, H. Bifunctional Networked Ag/AgPd Core/Shell Nanowires for the Highly Efficient Dehydrogenation of Formic Acid and Subsequent Reduction of Nitrate and Nitrite in Water. J. Mater. Chem. A 2018, 6, $4611-4616$

(41) Zheng, Z.; Tachikawa, T.; Majima, T. Plasmon-Enhanced Formic Acid Dehydrogenation Using Anisotropic Pd-Au Nanorods Studied at the Single-Particle Level. J. Am. Chem. Soc. 2015, 137, 948-957.

(42) Wu, B.; Lee, J.; Mubeen, S.; Jun, Y.-S.; Stucky, G. D.; Moskovits, M. Plasmon-Mediated Photocatalytic Decomposition of Formic Acid on Palladium Nanostructures. Adv. Opt. Mater. 2016, 4, 1041-1046.

(43) Bi, Q.-Y.; Lin, J.-D.; Liu, Y.-M.; Du, X.-L.; Wang, J.-Q.; He, H.Y.; Cao, Y. An Aqueous Rechargeable Formate-Based Hydrogen Battery Driven by Heterogeneous Pd Catalysis. Angew. Chem. Int. Ed. 2014, 53, 13583-13587.
(44) Ferry, J. G. Formate Dehydrogenase. FEMS Microbiol. Lett. 1990, 87, 377-382.

(45) Nikoobakht, B.; El-Sayed, M. A. Preparation and Growth Mechanism of Gold Nanorods (NRs) Using Seed-Mediated Growth Method. Chem. Mater. 2003, 15, 1957-1962.

(46) Liu, M.; Guyot-Sionnest, P. Mechanism of Silver(I)-Assisted Growth of Gold Nanorods and Bipyramids. J. Phys. Chem. B 2005, 109, 22192-22200.

(47) Tardy, B. L.; Yokota, S.; Ago, M.; Xiang, W.; Kondo, T.; Bordes, R.; Rojas, O. J. Nanocellulose-Surfactant Interactions. Curr. Opin. Colloid Interface Sci. 2017, 29, 57-67.

(48) Halas, N. J.; Lal, S.; Chang, W.-S.; Link, S.; Nordlander, P. Plasmons in Strongly Coupled Metallic Nanostructures. Chem. Rev. 2011, 111, 3913-3961.

(49) Grzelczak, M.; Pérez-Juste, J.; García de Abajo, F. J.; LizMarzán, L. M. Optical Properties of Platinum-Coated Gold Nanorods. J. Phys. Chem. C 2007, 111, 6183-6188.

(50) Hartland, G. V. Optical Studies of Dynamics in Noble Metal Nanostructures. Chem. Rev. 2011, 111, 3858-3887.

(51) Joo, J.; Uchida, T.; Cuesta, A.; Koper, M. T. M.; Osawa, M. The Effect of $\mathrm{pH}$ on the Electrocatalytic Oxidation of Formic Acid/ Formate on Platinum: A Mechanistic Study by Surface-Enhanced Infrared Spectroscopy Coupled with Cyclic Voltammetry. Electrochim. Acta 2014, 129, 127-136.

(52) Wu, J. T.; Wu, L. H.; Knight, J. A. Stability of NADPH: Effect of Various Factors on the Kinetics of Degradation. Clin. Chem. 1986, 32, 314-319.

(53) Chaykin, S.; King, L.; Gary Watson, J. The Reduction of $\mathrm{DPN}^{+}$ and $\mathrm{TPN}^{+}$with Sodium Borohydride. BBA-GEN Subjects 1966, 124, $13-25$.

(54) Begley, M. R.; Gianola, D. S.; Ray, T. R. Bridging Functional Nanocomposites to Robust Macroscale Devices. Science 2019, 364, No. eaav4299. 